Check for updates

1 Lancaster University, UK

2 University of British Columbia, Canada

Twitter: @dr_yanghu @yueqian_soc Cite this as: $B M J 2022 ; 376: 0256$ http://dx.doi.org/10.1136/bmj.0256 Published: 28 January 2022

\title{
Protecting older adults' mental health in the pandemic
}

Yang Hu, ${ }^{1}$ Yue Qian ${ }^{2}$

The recent rapid rise and ongoing high rates of covid-19 cases, driven by the spread of Omicron, are leading to an increase in hospital admissions and deaths among older adults. ${ }^{1}$ Due to their heightened vulnerability to covid-19, over the past two years, older people in many countries have been advised to adhere to household-centred containment measures such as household shielding. ${ }^{2}$ As a result, older people's face-to-face interactions have been substantially reduced during the pandemic, and their in-person contact with people from other households was largely replaced by virtual contact-for example, via telephone calls, texting, Zoom, FaceTime, and social media. ${ }^{3}$

Interactions with families and friends not living in the same household have long been known to help sustain people's mental health. These interactions are crucial to improving our mental health because they allow for the exchange of vital material, social, and emotional support.

While extensive efforts have been made to protect the older population from covid-19 infection, relatively less has been done to address the mental health crisis among older adults. Mental distress and loneliness have increased among older people across a number of countries, particularly during pandemic lockdowns. ${ }^{45}$ Research has linked an increase in loneliness and depressive symptoms among older adults to reduced in-person contact. Virtual interactions have not been found to benefit older people's mental health or reduce their loneliness. ${ }^{6}$ It is possible that without sufficient digital access, skills, and preparedness, some older adults experience digital stress, burnout, reluctance, and exclusion.

Accruing evidence on older adults' social interactions and mental health during covid-19 suggests a few important lessons. First, face-to-face inter-household contact-a part of everyday life that was often taken for granted before the pandemic -is a crucial mental health resource for older adults. Household-centred measures have featured prominently in covid-19 interventions, but have had unintended consequences for people's mental health. With the ongoing pandemic, it is therefore imperative to create safe ways for older people to meet up with families and friends from other households. In the long run, building resilient inter-household connections should become a key agenda item in public health campaigns.

Secondly, virtual contact as an alternative to face-to-face contact is not as beneficial to older people's mental health. The proliferation of digital communication and healthcare was already underway before covid-19, and the pandemic has considerably accelerated this process. However, there are limitations to a digital-only future compared to a digitally enhanced future of ageing and elderly care. ${ }^{6}$ Virtual contact would be better used to supplement, instead of replacing, face-to-face interactions.

As we respond to covid-19, learn from the pandemic, and prepare for future crises, it is high time that we prioritise older people's mental health needs. To do so, we must equip the ageing population with equitable access to digital technology and the skills required to use it. Accommodating the needs of diverse age groups should be a priority for the design of digital communication tools and healthcare platforms. And finally, as we live in an increasingly digitalised world, we must not forget the benefits of face-to-face interactions on our mental health.

\section{Competing interests: None declared.}

Office for National Statistics. Coronavirus (COVID-19) latest insights: Hospitals. https://www.ons.gov.uk/peoplepopulationandcommunity/healthandsocialcare/conditionsanddiseases/articles/coronaviruscovid19latestinsights/hospitals\#hospital-admissions-by-age

2 Ioannidis JPA. Precision shielding for COVID-19: metrics of assessment and feasibility of deployment. BMJ Glob Health 2021;6:e004614. doi: 10.1136/bmigh-2020-004614 pmid: 33514595

3 Freedman VA, Hu M, Kasper JD. Changes in Older Adults' Social Contact During the COVID-19 Pandemic, The Journals of Gerontology: Series B, 2021; gbab166, doi: 10.1093/geronb/gbab166

4 Webb L. COVID-19 lockdown: A perfect storm for older people's mental health. J Psychiatr Ment Health Nurs 2021;28:300-300. doi: 10.1111/jpm.12644. pmid: 32352621

5 Gloster AT, Lamnisos D, Lubenko J, etal. Impact of COVID-19 pandemic on mental health: An international study. PLoS One 2020;15:e0244809. doi: 10.1371/journal.pone.0244809. pmid: 33382859

6 Hu Y, Qian Y. COVID-19, Inter-household Contact and Mental Well-Being Among Older Adults in the US and the UK. Front Sociol 2021;6:714626. doi: 10.3389/fsoc.2021.714626. pmid: 34381838 